\title{
Analytical Solutions of Fukui-Ishibashi (FI) Model and Quick-Start (QS) Model
}

\author{
Shinji Kukida, Jun Tanimoto, Naoki Ikegaya, Aya Hagishimaors \\ Interdisciplinary Graduate School of Engineering Sciences, Kyushu University, Kasuga, Japan \\ Email: kukida.shinji@gmail.com
}

Received 7 May 2014; revised 7 June 2014; accepted 15 June 2014

Copyright (C) 2014 by authors and Scientific Research Publishing Inc.

This work is licensed under the Creative Commons Attribution International License (CC BY). http://creativecommons.org/licenses/by/4.0/

c) (i) Open Access

\begin{abstract}
Through straightforward deduction procedure, we explicitly show analytical solutions for both Fukui-Ishibashi (FI) model and Quick-Start (QS) model, which are fundamental deterministic Cellular Automaton (CA), applied to traffic flow.
\end{abstract}

\section{Keywords}

\section{Cellular Automaton, Analytical Solutions}

\section{Introduction}

For recent years, simulation study on traffic flow has been attracted much attention of physicists. Among wide variety of approaches, Cellular Automaton (CA), where vehicles are treated as discrete self-drive particles in an entirely discrete spatiotemporal system, are most heavily used because of its flexibility as well as robustness to apply various practical problems.

There have been proposed many traffic CA models so far. For example, Rule-184 [1], which was originally presented by Wolfram as a part of 256 elementary CA archetypes for general discussion, has been regarded as the simplest traffic model. Fukui-Ishibashi (FI) model [2] extended vehicle's speed $v$ more variable;

$v \in\left\{0,1, \cdots, V_{\max }\right\}$ instead of $v \in\{0,1\}$. Nagel-Schreckenberg (NS) model [3], which has been most heavily applied as a fundamental template model by many studies, considers random braking effect on the basis of FI model. Quick-start (QS) model [4] takes account of driver's anticipation effect. Slow-to-start (SlS) model [5] can consider inertia effect of vehicles. Base on NS model, Stochastic Nishinari-Fukui-Schadshneider (S-NFS) model [6] takes account of all those effects; random braking, quick-start as well as slow-to-start. Revised S-NFS model [7] improves random braking effect in order to reproduce synchronized flow depicted in Kerner's three phase theory [8]. Asymmetric Simple Exclusion Process (ASEP) [9] and Zero Range Process (ZRP) [10] are simpler CA models, thus have been used by some theoretical studies.

How to cite this paper: Kukida, S., Tanimoto, J., Ikegaya, N. and Hagishimaors, A. (2014) Analytical Solutions of Fukui-Ishibashi (FI) Model and Quick-Start (QS) Model. Journal of Applied Mathematics and Physics, 2, 691-697.

http://dx.doi.org/10.4236/jamp.2014.27076 
By previous works, analytical solutions of Rule-184, ASEP and ZRP have been derived, since flux-density relation can be fixed deterministically [11] [12]. Although both FI model and QS model never contain stochastic elements, analytical solutions for those two have not been known ever. This paper reports the result of it, which we are successfully able to deduce this time. .

\section{Analytical Solution of FI Model}

The update rule of FI model is as follows;

$$
\begin{aligned}
& \text { if }\left(\text { gap } \geq V_{\max }\right) \text { then }\left(v_{i}=V_{\max }\right), \\
& \text { if }\left(\text { gap }<V_{\max }\right) \text { then }\left(v_{i}=\text { gap }\right),
\end{aligned}
$$

where gap means the number of unoccupied sites in front of vehicle $i, V_{\max }$ means the maximum velocity and $v_{i}$ means the velocity of vehicle $i$. It is obvious from Figure 1 that the critical density, making traffic flux maximum, is $k_{c r i}=\frac{1}{V_{\max }+1}$. Thus, the maximum flux is drawn by $Q_{\max }=\frac{V_{\max }}{V_{\max }+1}$.

Let us prove that the fundamental diagrams by FI model can be described as an asymmetrical tent-type polygonal line functions as below.

Proposition 1

When $k \leq k_{c r i}$, it is trivial that the relation between density and flux must be a linear function originated from $(k, Q)=(0,0)$ with slope $V_{\max }$.

When $k \geq k_{c r i}$, let us evaluate the relation between density and flux as follows. Figure 2 shows an example of flow state when $k \geq k_{\text {cri }}$. Schematic implication and definition of average velocity, we know;

$$
(\text { averagevelocity })=(\text { total sum of all vehicles' velocity }) /(\text { number of vehicles })
$$

and it is also trivial;

$$
\text { ( totalsum of all vehicles' velocity })=(1-k) L,
$$

where $L$ is system length. Hence,

$$
\text { (average velocity) }=L(1-k) /(L \cdot k)
$$

Therefore, flux $Q(k)$ is;

$$
Q(k)=1-k,
$$

which explicitly implies a linear function of $k$. This negative slope line obviously crosses both critical state and complete jam state; $\left(\frac{1}{V_{\max }+1}, \frac{V_{\max }}{V_{\max }+1}\right)$ and $(1,0)$.

(QED)

According to Proposition 1, the fundamental diagram can be described as an asymmetric tent-type function, consisting of two liner functions. The above-deduced critical density and maximum flux is the vertex of this asymmetric tent-type function.

As the next step, we should discuss whether the function consisting of two linear lines can be expressed by a single expression

Proposition 2

What we expect is that the function consisting of two linear lines can be expressed by a single expression; $f: Q=f(k)$ (Figure 3(a)). Shifting the function in the direction of $k$ by $-p$, we obtain $f^{\prime}: Q=f(k+p)$ (Figure 3(b)).

Meanwhile, two equations to touch at $(k, Q)=(0, q) ; f_{1}$ and $f_{2}$, shown in Figure 3(b) can be expressed as below; 


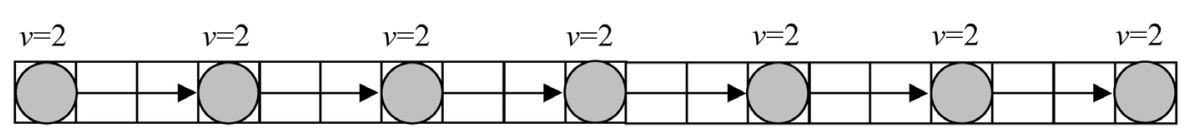

(a) $k=k_{c r i}$ and $V_{\max }=2$

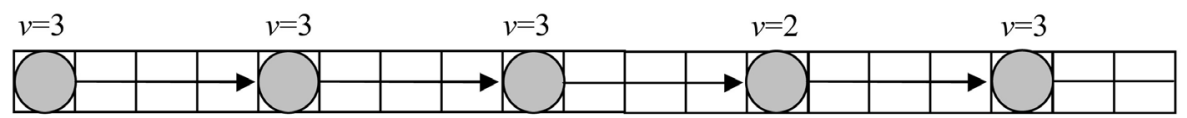

(b) $k=k_{c r i}$ and $V_{\max }=3$

Figure 1. Flow states explaining for FI model.

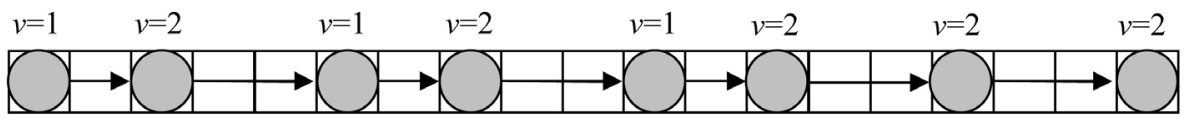

(a) $k \geq k_{\text {si }}$ and $\mathrm{V}_{\max }=2$

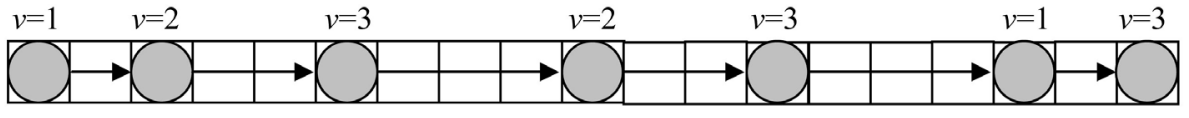

(b) $k \geq k_{c r i}$ and $\mathrm{V}_{\max }=3$

Figure 2. Flow states explaining for FI model in case if $k \geq k_{c r i}$.

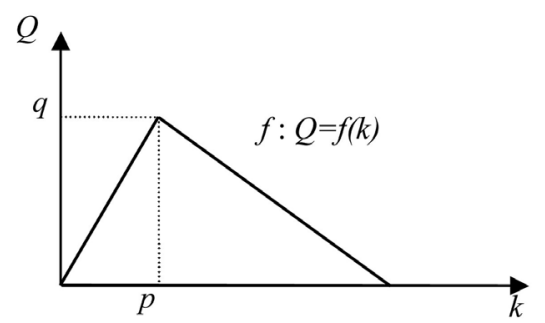

(a)

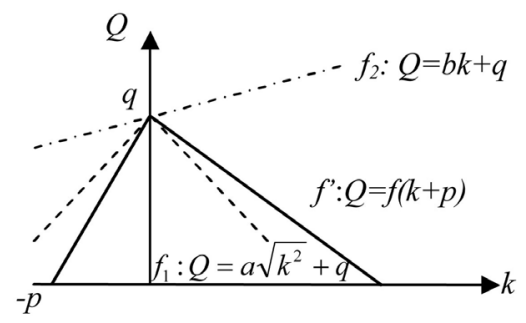

(b)

Figure 3. Asymmetric tent-type function.

$$
\begin{gathered}
f_{1}: Q=a \sqrt{k^{2}}+q . \\
f_{2}: Q=b k+q .
\end{gathered}
$$

The expression of $f_{1}$ is determined because it must be symmetric against $Q$ axis. By superposing those two liner functions, we obtain the asymmetric tent-type functionas below;

$$
f^{\prime}: Q=f(k+p) \equiv f_{1}+f_{2}-q,
$$

By substituting Equations (1) \& (2), we obtain:

$$
f(k+p)=a \sqrt{k^{2}}+b k+q .
$$

When the branch of square root is taken into consideration, it is as follows; if $k<0$ then,

$$
f(k+p)=(-a+b) k+q \equiv D_{1} k+q,
$$

if $k>0$ then,

$$
f(k+p)=(a+b) k+q \equiv D_{2} k+q
$$

We know; $(p, q)=\left(\frac{1}{V_{\max }+1}, \frac{V_{\max }}{V_{\max }+1}\right)$ and $D_{1}=V_{\max }, D_{2}=-1$. 
By substituting above conditions into Equations (4) \& (5), we get;

$$
a=-\frac{V_{\max }+1}{2}, b=\frac{V_{\max }-1}{2} .
$$

By substituting these into Equation (2), we obtain the following.

$$
f(k+p)=-\frac{V_{\max }+1}{2} \sqrt{k^{2}}+\frac{V_{\max }-1}{2} k+q .
$$

As consequence, $f(k)$ is;

$$
f(k)=-\frac{V_{\max }+1}{2} \sqrt{(k-p)^{2}}+\frac{V_{\max }-1}{2}(k-p)+q .
$$

Namely; we obtain;

$$
\therefore Q=-\frac{V_{\text {max }}+1}{2} \sqrt{\left(k-\frac{1}{V_{\text {max }}+1}\right)^{2}}+\frac{V_{\text {max }}-1}{2}\left(k-\frac{1}{V_{\text {max }}+1}\right)+\frac{V_{\text {max }}}{V_{\text {max }}+1}
$$

By rearranging, analytical solution of FI model can be derived as follows;

$$
Q=\frac{V_{\max }}{1+V_{\max }}-\left\{\frac{1+V_{\max }}{2} \sqrt{\left(k-\frac{1}{1+V_{\max }}\right)^{2}}+\frac{1-V_{\max }}{2}\left(k-\frac{1}{1+V_{\max }}\right)\right\},
$$

where $Q$ means the flux and $k$ means density, respectively.

Figure 4 shows the fundamental diagrams by Equation (10) when $V_{\max }=1,2,3,4$.

\section{Analytical Solution of QS Model}

The update rule of QS model is as follows;

If there is an empty site in forward $S$-sites then the focal vehicle moves.

Where $S$ means the number of sites that a vehicle foresees for quick-start. It is obvious from Figure 5 that the critical density is $k_{c r i}=\frac{S}{S+1}$. Thus, the maximum flux is drawn by $Q_{\max }=\frac{S}{S+1}$, because $v=1$. Like FI model, Let us prove that the fundamental diagrams by QS model can be described as an asymmetrical tent-type poly-gonal line functions as below.

Proposition 3

When $k \leq k_{c r i}$, it is trivial that the relation between density and flux must be a linear function originated from $(k, Q)=(0,0)$ with slope $V_{\max }=1$.

When $k \geq k_{\text {cri }}$, let us evaluate the relation between density and flux as follows. Figure 6 shows an example of flow state when $k \geq k_{c r i}$. Schematic implication and definition of average velocity, we know;

$$
\begin{aligned}
(\text { averagevelocity }) & =(\text { total sum of all vehicles' velocity }) /(\text { number of vehicles }) \\
& =(\text { number of vehicles that can move }) /(\text { number of vehicles })
\end{aligned}
$$

Observing Figure 6, we notice that vehicles staying in $S$ sites behind each empty site can move. Thus;

$$
\text { (number of vehicles that can move) }=L(1-k) S \text {, }
$$

and;

$$
(\text { averagevelocity })=L(1-k) S, /(L \cdot k)
$$

Therefore, flux $Q(k)$ is;

$$
Q(k)=(1-k) S,
$$

which explicitly implies a linear function of $k$. This negative slope line obviously crosses both critical state and 


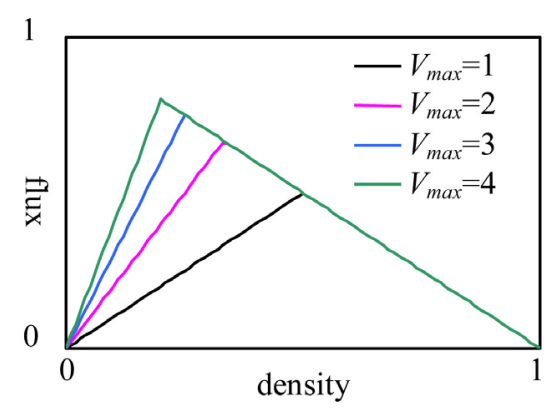

Figure 4. The fundamental diagram by Equation (9).

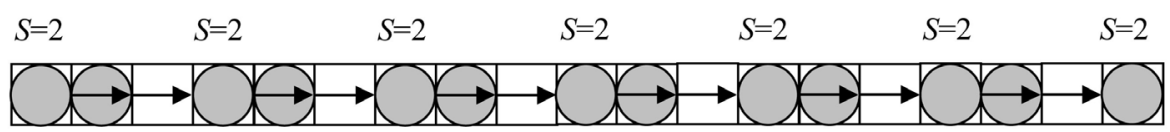

(a) $k \geq k_{c i}$ and $S=2$

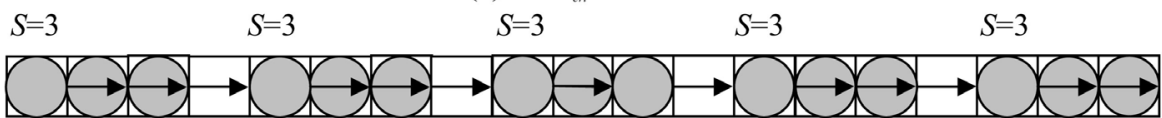

(b) $k \geq k_{c r}$ and $S=3$

Figure 5. Flow states explaining for QS.

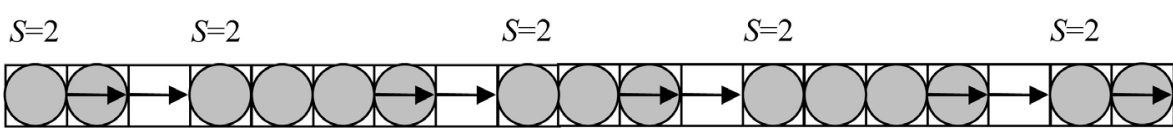

(a) $k \geq k_{\text {cri }}$ and $\mathrm{S}=2$

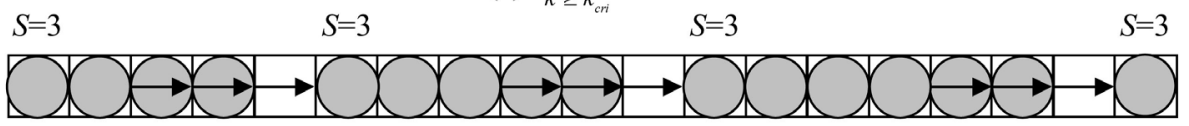

(b) $k \geq k_{c r i}$ and $\mathrm{S}=3$

Figure 6. Flow states explaining for QS model $l$ in case if $k \geq k_{c r i}$.

complete jam state; $\left(\frac{S}{S+1}, \frac{S}{S+1}\right)$ and $(1,0)$.

(QED)

Proposition 3 enables us to draw that the fundamental diagram can be described as an asymmetric tent-type function, consisting of two liner functions. The above-deduced critical density and maximum flux is the vertex of this asymmetric tent-type function.

Like the case of FI model, as the next step for the discussion, we should note how Proposition 2 leads that the function consisting of two linear lines can be expressed by a single expression

Namely, we know; $(p, q)=\left(\frac{S}{S+1}, \frac{S}{S+1}\right)$ and $D_{1}=1, D_{2}=-S$.

By substituting above conditions into Equations (4) \& (5), we get;

$$
a=-\frac{S+1}{2}, b=-\frac{S-1}{2}
$$

By substituting these into Equation (2), we obtain the following.

$$
f(k+p)=-\frac{S+1}{2} \sqrt{k^{2}}-\frac{S-1}{2} k+q
$$

Therefore, $f(k)$ is; 


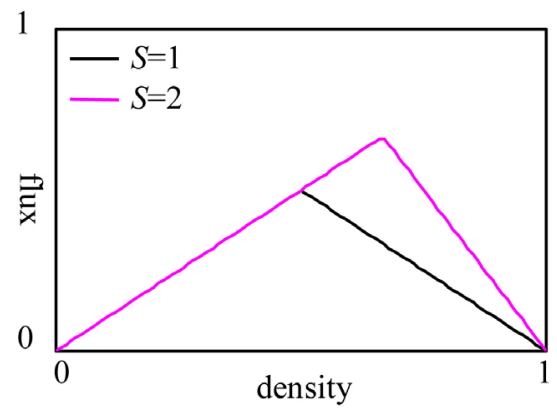

Figure 7. The fundamental diagram by Equation (13).

$$
f(k)=-\frac{S+1}{2} \sqrt{(k-p)^{2}}-\frac{S-1}{2}(k-p)+q .
$$

Finally, we get;

$$
\therefore Q=-\frac{S+1}{2} \sqrt{\left(k-\frac{S}{S+1}\right)^{2}}-\frac{S-1}{2}\left(k-\frac{S}{S+1}\right)+\frac{S}{S+1}
$$

By rearranging, analytical solution of QS model is derived as follows;

$$
Q=\frac{S}{S+1}-\left\{\frac{S+1}{2} \sqrt{\left(k-\frac{S}{S+1}\right)^{2}}+\frac{S-1}{2}\left(k-\frac{S}{S+1}\right)\right\}
$$

Figure 7 shows the fundamental diagrams by Equation (13) when $S=1,2$.

\section{Conclusions}

We explicitly reported analytical solutions fort FI model and QS model.

Although the two are important models for depicting basic traffic features, analytical solutions for those two have not been known ever.

Helped by the fact that FI model and QS model never contain stochastic elements, our process to deduce was simple and straightforward.

\section{Acknowledgements}

This study was partially supported by a Grant-in-Aid for Scientific Research by the Japan Society for the Promotion of Science, awarded to Prof. Tanimoto (\#25560165), Tateishi Science and Technology Foundation. We would like to express our gratitude to these funding sources.

\section{References}

[1] Wolfram, S. (1986) Theory and Applications of Cellular Automata. World Scientific, Singapore.

[2] Fukui, M. and Ishibashi, Y. (1996) Traffic Flow in 1D Cellular Automaton Model including Cars Moving with High Speed. Journal of the Physical Society of Japan, 65, 1868-1870. http://dx.doi.org/10.1143/JPSJ.65.1868

[3] Nagel, K. and Schreckenberg, M. (1992) A Cellular Automaton Model for Freeway Traffic. Journal de Physique I, 2, 2221. http://dx.doi.org/10.1051/jp1:1992277

[4] Barlovic, R., Santen, L., Schadschneider, A. and Schreckenberg, M. (1998) Metastable State in Cellular Automata for Traffic Flow. European Physical Journal B, 5, 793-800. http://dx.doi.org/10.1007/s100510050504

[5] Nishinari, K. and Takahashi, D. (2000) Multi-Value Cellular Automaton Model for Freeway Traffic. Journal of Physics A, 33, 7709. http://dx.doi.org/10.1088/0305-4470/33/43/304

[6] Sakai, S., Nishinari, K. and Iida, S. (2006) A New Stochastic Cellular Automaton Model on Traffic Flow and Its Jamming Phase Transition. Journal of Physics A: Mathematical and General, 39, 15327. http://dx.doi.org/10.1088/0305-4470/39/50/002 
[7] Kokubo, S., Tanimoto, J. and Hagishima, A. (2011) A New Cellular Automata Model Including a Decelerating Damping Effect to Reproduce Kerner's Three-Phase Theory. Physica A: Statistical Mechanics and Its Applications, 390, 561-568. http://dx.doi.org/10.1016/j.physa.2010.10.027

[8] Kerner, B.S. and Klenov, S.L. (2009) Phase Transitions in Traffic Flow on Multilane Roads. Physical Review E, 80, Article ID: 056101. http://dx.doi.org/10.1103/PhysRevE.80.056101

[9] Derrida, B., Evans, M.R., Hakim, V. and Pasquier, V. (1993) Exact Solution of a1D Asymmetric Exclusion Model Using a Matrix Formulation. Journal of Physics A: Mathematical and General, 26, 1493. http://dx.doi.org/10.1088/0305-4470/26/7/011

[10] O’Loan, O.J., Evans, M.R. and Cates, M.E. (1998) Jamming Transition in a Homogeneous One-Dimensional Systems: The Bus Route Model. Physical Review E, 58, 1404. http://dx.doi.org/10.1103/PhysRevE.58.1404

[11] Schadschneider, A. and Schreckenberg, M. (1993) Cellular Automaton Models and Traffic Flow, Journal of Physics A: Mathematical and General, 26, L679. http://dx.doi.org/10.1088/0305-4470/26/15/011

[12] Klauck, K. and Shadshneider, A. (1999) On the Ubiquity of Matrix-Product States in One-Dimensional Stochastic Processes with Boundary Interactions. Physica A, 271, 102-117. http://dx.doi.org/10.1016/S0378-4371(99)00176-4 
Scientific Research Publishing (SCIRP) is one of the largest Open Access journal publishers. It is currently publishing more than 200 open access, online, peer-reviewed journals covering a wide range of academic disciplines. SCIRP serves the worldwide academic communities and contributes to the progress and application of science with its publication.

Other selected journals from SCIRP are listed as below. Submit your manuscript to us via either submit@scirp.org or Online Submission Portal.
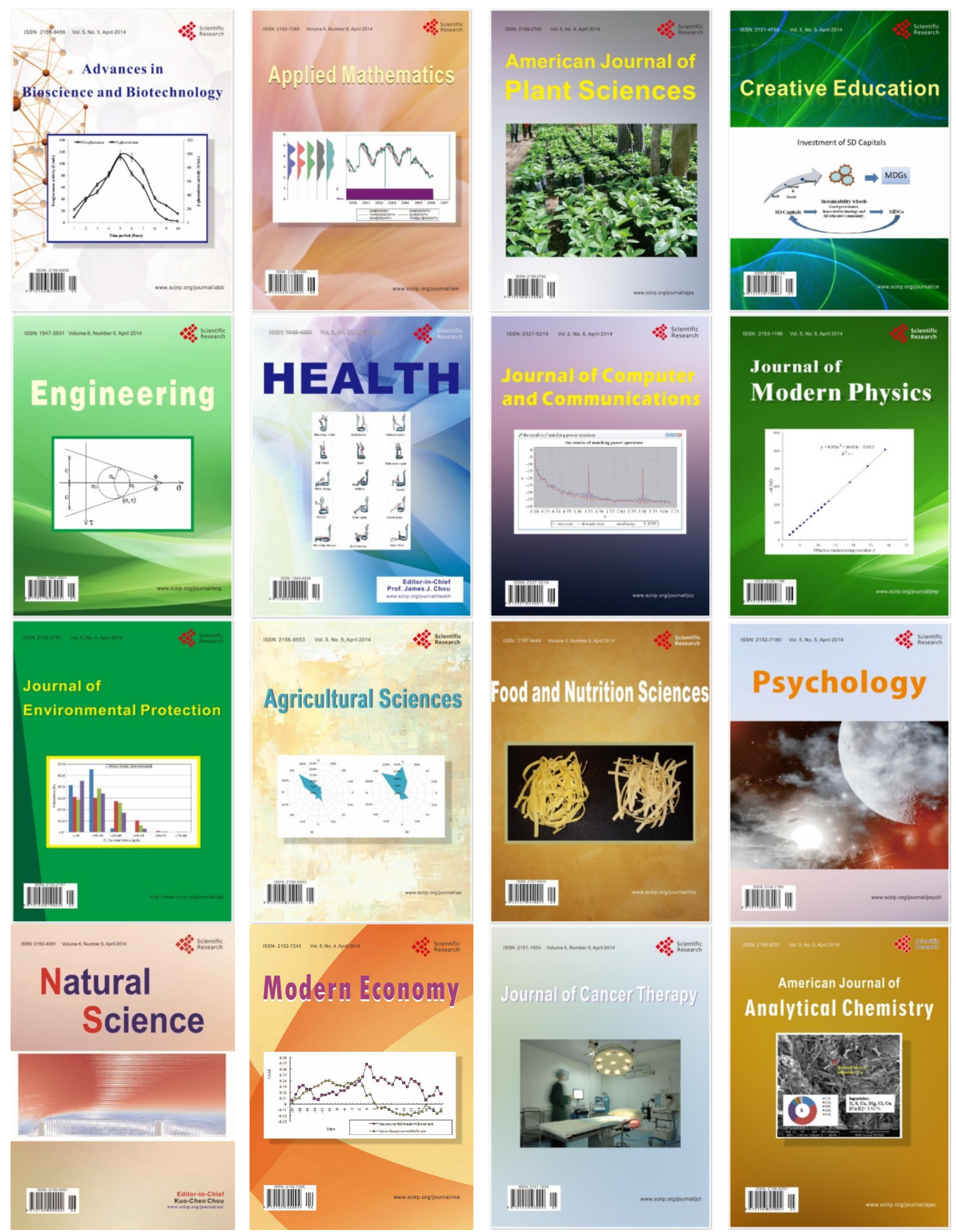